\title{
The role of the study of exercise in defining the Frontier of Integrative Biology
}

\author{
Gary A. Iwamoto ${ }^{1,2 *}$ \\ ' Department of Comparative Bioscience, Molecular and Integrative Physiology, University of Illinois at Urbana-Champaign, Urbana, IL, USA \\ 2 Neuroscience Program, University of Illinois at Urbana-Champaign, Urbana, IL, USA \\ *Correspondence: iwamoto@uiuc.edu
}

Of all the fields that comprise the physiological sciences, exercise is one of the hardest to categorize even though it is a common everyday experience for nearly everyone whether it is a formal exercise program or simply walking from one place to another. This familiarity makes the field understandable to the average person, but this familiarity also sometimes leads to an oversimplification of the complexity of the investigation of the physiological response to exercise. This is further complicated by the idea, as Tipton and Franklin (2006) point out, that exercise has different meanings for different people and is thus inherently diverse in terms of what one may think it consists of, as well as diverse in the lines of scientific inquiry that concern it.

It is sometimes forgotten that fundamental definitions of life have exercise implicit in their very wording and includes "the energy that enables organisms to grow, reproduce, absorb and use nutrients, and evolve, and, in some organisms, to achieve mobility, express consciousness, and demonstrate a voluntary use of the senses" (Myers, 2009). Put yet another way: "The property or quality that distinguishes living organisms from dead organisms and inanimate matter, manifested in functions such as metabolism, growth, reproduction, and response to stimuli or adaptation to the environment originating from within the organism" (Spitz, 2007). The key ideas here are mobility, and response to stimuli or adaptation - both intrinsic to the study of exercise. At the risk of overstating the obvious, Astrand and Rodahl's (1986) classic Textbook of Work Physiology gently reminds the reader that while most physiology considers the organism at rest, the factor that sets exercise physiology apart is that it considers the organism in the performance of physical activity.

These are comparatively simple ideas compared to the diversity of subject matter found within exercise physiology. Being inclusive of the subject matter is difficult because exercise by its nature can involve nearly the entire organism in term its response and adaptation. While the roles of the musculoskeletal, cardiovascular, respiratory, and nervous systems are obvious, even the reproductive related hormones have roles to play in the expression of exercise. Furthermore, reproductive function is certainly affected by various types of exercise training. Perhaps the most recent volume of the Handbook of Physiology (Rowell and Shepherd, 1996) came the closest to defining what is entailed by exercise physiology, by simply indicating that exercise constituted the "regulation and integration of multiple systems". It is thus incumbent upon each investigator to know that exercise physiology involves the study of many if not all of these systems. In most cases, the work of the more specialized investigators of individual systems will find application of their research within the exercise field.

The questions facing the field of exercise physiology are ones which have a certain universality to the subject matter and often the evidence from exercise related experiments provides valuable clues that aid in answering questions fundamental to the study of the systems themselves. While there is the inherent wide range of subject matter, for some questions the connection to exercise is straightforward:

Within the physical act of exercise one would include musculoskeletal and motor control. In the latter case, one would have to include the investigation of the significance of muscle fiber and motor unit types. More recent studies have been begun to study changes in the musculoskeletal system over the lifespan, as aging presents its own set of unique questions to be answered. In this context, one might ask questions such as what causes sarcopenia or is there any preferential loss of certain fiber types with aging. Still other questions involve understanding neuromuscular control such as how many synergies exists for even basic motor tasks such as reaching, grasping, and walking? Investigators sometimes forget that this area of study is a part of exercise physiology just as surely as the classic metabolic studies remain as an essential part of our knowledge base.

Within the other aspects of the neurosciences, is it possible for us to ever really know what aspects of connectivity and sequencing of the cell activity within the central neural control of locomotor activity, circulation, and respiration drive the exercise response? Further, what is the nature of the spinal cord central pattern generator of locomotion and its respiration counterpart within the higher control centers? What is/are "central command" site(s) within the central nervous system? The description of these neural cells and their arrangement within the brain and spinal cord awaits elucidation. In yet another context, where does volitional motor activity come from and to what extent is this linked to the control of other systems? While therapeutic application of this knowledge in terms of motor activity is still far becoming a part of the standard practice, the understanding of these systems may someday make clinical applications possible. To know these answers will, in all likelihood, allow us to know the secret of volition itself.

In the context of neural control, one must also include the changing nature of what constitutes fatigue. One should not forget that it was not so long ago that peripheral mechanisms dominated the field with only a grudging acknowledgment of the possibility of central fatigue. Yet, even now, we have not solved the problem of fatigue within cases such as McArdle's syndrome in which the metabolite, lactic acid, apparently does not play a role. Conversely, the fatigue that accompanies many neurological disorders (such as various conditions involving the cerebellum) represents another mystery to 
be solved. It is clear that both central and peripheral mechanisms must be involved in the fatigue in individuals with these diseases but how these mechanisms interact remain largely to be determined.

We are only now beginning to appreciate that benefits of exercise extend far beyond simple aerobic fitness. Among these benefits, one must include the extent to which exercise improves higher brain function and its effects as the stimulation of the immune system. Although significant progress in these areas has been made in recent years, many questions remain to be answered. It is worth recalling at this point that the study of these systems hinges on the knowledge of other areas such as the circulatory and endocrine systems as well as their neural control. Thus, once again we hear the recurring echo emphasizing that integrative control of multiple systems cannot be forgotten.

While these aspects may represent the "complex", even longstanding and apparently simple ideas, such as the idea that the vasodilator responses in exercising muscle resulted from significant neural cholinergic mechanisms (a concept that has been in textbooks for many years), will have to be revised in the near future. It is now apparent, that this old concept of a cholinergic mediated vasodilation is not true - but the story is still not complete. The identification of the actual mechanism (s) for the well-established exercise induced increase in skeletal muscle blood flow awaits elucidation.

It should not be forgotten that understanding how the living organism responds and adapts to exercise under idealized control conditions could provide only a limited insight as to how the physiological to response to exercise under changing environmental conditions. Thus, many aspect of physiology that usually fall under the heading of "environmental physiology" also should be included as part of exercise physiology. Environmental variations such changing in temperature and altitude, as well as the hyper- or hypo-dynamic conditions can profoundly affect the response to exercise. Hypodynamic (disuse) conditions are essentially the reverse of exercise as one might generally conceive it and include another of the universal experiences bedrest. Under these special conditions, an entirely new set of data would be expected across all systems.
Certainly one of the overwhelming considerations for the future of much biological inquiry is the application of molecular techniques. This is no less true for exercise physiology than any other branch of physiological investigations. We are at an interesting time, a time in which molecular techniques are acquiring the precision necessary for physiological applications. Thus, it was not long ago that these techniques only had the precision adequate to analyze signals at the organ or tissue level, but they have been sufficiently improved for analysis at the level of the single cells and when applicable subcellular organelles. The application of genetic array technology can now be applied to exercise physiology, across all disciplines and in particular tissues that are not homogenous.

However, despite the seductive power of reductionism that utilizes these molecular approaches, one must not lose sight that an understanding of the exercise physiology ultimately requires a comprehensive knowledge of the integrated response among multiple organ systems in the intact organism. Although the pioneering studies were completed many years ago, these studies nonetheless still provide the base upon which all future investigations will be based. We will continue to use and to refine these benchmarks (such as oxygen uptake and quantitative exercise in the whole animal) as new technologies and new questions emerge for years to come.

With regards investigation of function of the whole organism, animal models greatly facilitate the study of exercise. Animals should not, however, be thought of in just the narrow capacity as models for humans in invasive chronic instrumentation studies. It has not been that long ago that the study of animals as athletes became commonplace with the obvious connection that their study in exercise physiology benefited both humans and animals. More specialized investigations of animal "athletes", ranging from racing greyhounds to the various equestrian sports, will most certainly make substantial contributions to advancement of exercise.

The rationale for the application of exercise in medical practice should be constantly reevaluated as more and better information from basic science (in both human and animals) is accumulated. "Evidence based medicine" will continue to be of the utmost importance. In many of the cases mentioned above (as well others that have not been described), there are clear health benefits that result from basic research. This simple statement would seem to be self evident to most in the workers in the exercise physiology field.

In fact, many of the leaders of the field have already suggested that the "exercise prescription" as applied in the clinic, needs modification and should be rewritten (Green et al., 2008) to reflect the most recent evidence obtained from basic research - some of which we hope to present in this journal. While these authors focused primarily on cardiovascular benefits of exercise, it should be stressed that the concept of an "exercise prescription" itself has become increasing supported by evidence obtained from basic research (Billman, 2002; Joyner and Green, 2009). It was only a short time ago that the therapeutic potential of exercise was largely based upon "educated" guesses. We are now beginning to understand the physiology upon which a rational exercise prescription can be based. It is clear that one of the unanswered questions would be how exercise actually reverses endothelial dysfunction and its attendant effects on the cardiovascular system. Further, how does the autonomic nervous system contribute to this beneficial effect of exercise? Joyner and Green (2009) emphasize that the combination of high sympathetic outflow, reduced vasodilator function and cardiovascular disease form a vicious cycle that amplifies over time, a hypothesis that further demonstrates that the exercise response involves multiple systems.

There is perhaps still an even greater challenge confronting exercise physiology than those scientific challenges highlighted in the preceding sections of this essay; namely, a shift in the emphasis in exercise physiology training programs from the generation of new knowledge to the dissemination of this knowledge. In recent years, a sentiment has arisen that since "everything that is important about exercise physiology is known"; attention should be diverted away from the basic science of exercise to increased emphasis on promotion of exercise. The individuals who support this view ignore the basic fact that not all the important questions have been answered, and further that basic research provides the evidence that gives the exercise sciences their legitimacy as serious subjects, as well as allowing for its application to medical practice. The discovery of new 
information remains crucial for the advancement of exercise physiology and in the final analysis should be included in any training program that carries this name.

Thus, part of the challenge facing exercise physiology is to prepare the next generation of scientists so that they can continue the search for new knowledge in integrative physiology. It is one of the principles aims of this journal to provide a forum that will support these investigators in their quest for knowledge at the "frontiers" of exercise physiology.

\section{ACKNOWLEDGMENTS}

I am indebted to Dr. Roger Enoka, professor and chair of the Department of Integrative Physiology, University of Colorado, Dr. Michael Joyner professor, Department of
Anesthesiology, Mayo Medical School and Dr. Marc Kaufman professor, Heart and Vascular Institute of Pennsylvania State University Medical School for assisting with this essay.

\section{REFERENCES}

Astrand, P.-O., and Rodahl, K. (1986). Textbook of Work Physiology. Singapore: McGraw-Hill.

Billman, G. (2002). Aerobic exercise conditioning: a nonpharmacological antiarrhythmic intervention. J. Appl. Physiol. 92, 446-454.

Green, D. J., O'Driscoll, G., Joyner, M. J., and Cable, N. T. (2008). Exercise and cardiovascular risk reduction: time update the rationale for exercise? J. Appl. Physiol. $105,766-768$.

Joyner, M. J., and Green, D. J. (2009). Exercise protects the cardiovascular system: effects beyond traditional risk factors. J. Physiol. (Lond.). 587, 5551-5558.

Myers, T.Ed. (2009). Mosby's Medical Dictionary, 8th Edn. St. Louis: Mosby Elsevier.

Rowell, L. B., and Shepherd, J. T. (1996). Handbook of Physiology Section 12 Exercise: Regulation and
Integration of Multiple Systems. New York: Oxford University Press.

Spitz, S. (Project ed.) (2007). The American Heritage Medical Dictionary. Boston: Houghton Mifflin Company.

Tipton, C. M., and Franklin, B. A. (2006). “The language of exercise," in ACSM's Advanced Exercise Physiology, ed. C. M. Tipton (Baltimore, Philadelphia: Williams and Wilkins), 3-10.

Received: 08 October 2010; accepted: 08 October 2010; published online: 25 November 2010.

Citation: Iwamoto GA(2010) The role of the study of exercise in defining the Frontier of Integrative Biology. Front. Physio. 1:142. doi: 10.3389/fphys.2010.00142

This article was submitted to Frontiers in Exercise Physiology, a specialty of Frontiers in Physiology.

Copyright $(0) 2010$ Iwamoto. This is an open-access article subject to an exclusive license agreement between the authors and the Frontiers Research Foundation, which permits unrestricted use, distribution, and reproduction in any medium, provided the original authors and source are credited. 